\title{
Erratum a: ATA vs EU-TIRADS vs AACE/ACE/AME: criteri ecografici tiroidei a confronto
}

\author{
T. Rago ${ }^{1} \cdot$ S. Manfrini ${ }^{2} \cdot$ S. Briganti ${ }^{2} \cdot$ A. Palermo ${ }^{2}$
}

Pubblicato online: 5 dicembre 2018

(c) Springer Nature Switzerland AG 2018

Erratum a: L'Endocrinologo (2018) 19(4): 210-212 https://doi.org/10.1007/s40619-018-00468-y

In seguito alla pubblicazione dell'articolo, sono stati rilevati errori e inesattezze.

Ci scusiamo coi lettori e riportiamo di seguito le correzioni:

\section{Lista degli autori}

La lista degli autori dell'articolo pubblicato non è corretta, di seguito l'elenco corretto:

\section{T. Rago ${ }^{1}$, S. Manfrini ${ }^{2}$, S. Briganti ${ }^{2}$, A. Palermo ${ }^{2}$}

1 Dipartimento Attività Integrata Specialità Mediche, U.O. di Endocrinologia I, Azienda Ospedaliero-Universitaria di Pisa, Università di Pisa; Pisa, Italia

2 Struttura Complessa di Endocrinologia del Policlinico dell'Università Campus Biomedico, Roma, Italia.

2. Paragrafo "Raccomandazioni per l'esecuzione di FNA nei noduli tiroidei

\section{Linee guida ATA}

- Nodulo benigno: no FNA.

- Nodulo a rischio molto basso: osservazione; FNA nei noduli di dimensioni $\geq 2 \mathrm{~cm}$

La versione online dell' articolo originale può essere trovata al https://doi.org/10.1007/s40619-018-00468-y.

T. Rago

rago@endoc.med.unipi.it

1 Dipartimento Attività Integrata Specialità Mediche, U.O. di Endocrinologia I, Azienda Ospedaliero-Universitaria di Pisa, Università di Pisa, Pisa, Italia

2 Struttura Complessa di Endocrinologia del Policlinico dell'Università Campus Biomedico, Roma, Italia
- Nodulo a rischio basso: FNA nei noduli $\geq 1,5 \mathrm{~cm}$

- Nodulo a rischio intermedio: FNA nei noduli $>1 \mathrm{~cm}$

- Nodulo ad alto rischio: FNA nei noduli $>1 \mathrm{~cm}$ (Tabella 1 )

\section{Linee guida AACE/ACE/AME:}

- Nodulo a rischio basso: noduli spongiformi o prevalentemente cistici senza caratteri ecografici sospetti, raccomandato FNA solo quando i noduli sono $>2 \mathrm{~cm}$, se aumentano di dimensioni o sono associati una storia di alto rischio prima della chirurgia o terapia ablativa mini-invasiva. I noduli a basso rischio $<5 \mathrm{~mm}$ dovrebbero essere monitorati, indipendentemente dai caratteri ecografici.

- Nodulo a rischio intermedio: FNA nei noduli $>2 \mathrm{~cm}$.

- Nodulo a rischio alto: FNA nei noduli $\geq 1 \mathrm{~cm}$. Nei noduli ad alto rischio di dimensioni tra 5-10 mm, in accordo con il paziente, considerare l'osservazione clinica o FNA. (Tabella 1) FNA è raccomandata

- nei noduli sottocapsulari o lesioni paratracheali,

- in presenza di linfonodi sospetti o diffusione extratiroidea

- storia personale o familiare carcinoma della tiroide

- pregressa irradiazione testa -collo

- altri quadri clinici sospetti (ad es. comparsa di disfonia)

\section{Paragrafo "Raccomandazioni per eseguire l'FNA"}

- EU-TIRADS 1: FNA no raccomandato

- EU-TIRADS 2: FNA no raccomandato

- EU-TIRADS 3: FNA raccomandato se nodulo $>2 \mathrm{~cm}$

- EU-TIRADS 4: FNA raccomandato se nodulo $>1,5 \mathrm{~cm}$

- EU-TIRADS 5: FNA raccomandato se nodulo $>1 \mathrm{~cm}$, nei noduli di dimensioni $>1 \mathrm{~cm}$ raccomandato FNA o sorveglianza attiva 


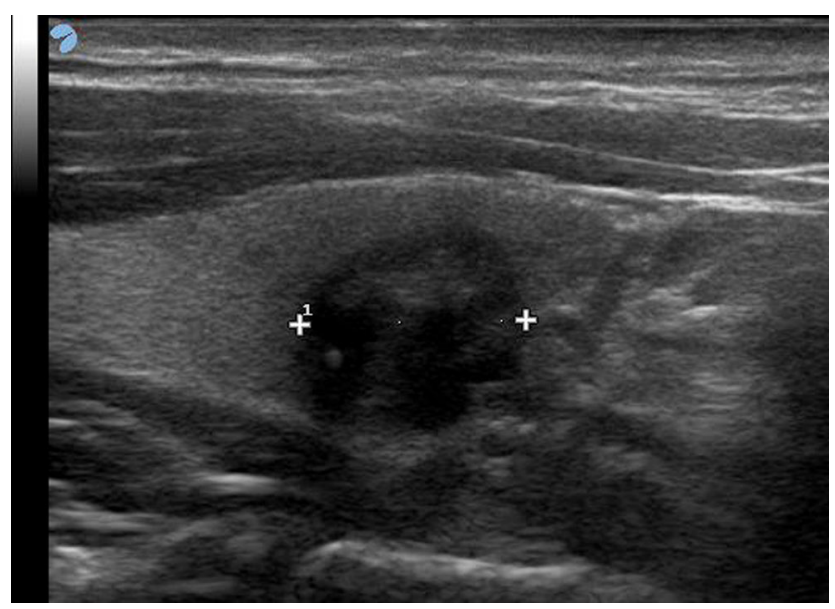

Immagine ecografica (sezione longitudinale) che documenta in un lobo tiroideo di normali dimensioni la presenza di un nodulo solido, ipoecogeno, disomogeneo, con lacune, margini irregolari, sfrangiati $6 \times 8 \times 11 \mathrm{~mm}$.

Secondo le classificazioni descritte, questo nodulo dovrebbe essere considerato a rischio alto. Il paziente è stato sottoposto a tiroidectomia per una citologia sospetta. L'esame istologico ha confermato la diagnosi di Ca papillare.

\section{Conflitto di interesse}

Gli Autori T. Rago, S. Manfrini, S. Briganti e A. Palermo dichiarano di non avere conflitti di interesse. 